Supporting Information

\title{
Ultraviolet Radiation Enhances the Toxicity of Deepwater Horizon Oil to Mahi-mahi (Coryphaena hippurus) Embryos
}

Matthew Alloy ${ }^{\dagger}$, David Baxter ${ }^{\dagger}$, John Stieglitz ${ }^{\ddagger}$, Edward Mager $^{\ddagger}$, Ronald Hoenig ${ }^{\ddagger}$, Daniel Benetti ${ }^{\ddagger}$, Martin Grosell $^{\ddagger}$, James Oris ${ }^{\S}$, and Aaron Roberts* ${ }^{\dagger}$

${ }^{\dagger}$ University of North Texas, Department of Biological Sciences 1155 Union Circle \#305220

Denton, TX 76203, USA

‡ Division of Marine Biology and Fisheries, Rosenstiel School of Marine and Atmospheric Sciences, University of Miami, Miami, FL 33149-1098

${ }_{\S}^{\S}$ Miami University, Department of Biology, 700 East High Street, Oxford, OH 45056, USA

Number of pages: 4

Number of tables: 2

\section{Supporting information contents}

Table S1. Concentrations of individual PAHs in stock solutions.

$\mathrm{S} 2-\mathrm{S} 3$

Table S2. The individual PAH detection limits used in each stock analysis.

S3 - S4 
Tables1.

Concentrations of individual polycyclic aromatic hydrocarbons (PAHs) in stock solutions. Values below the quantification limit are listed as "-“. Bold indicates one of the 14 photodynamic PAHs.

Water accommodated fraction tock $\mathrm{tPAH}_{50}$ composition $(\mu \mathrm{g} / \mathrm{L})$

\begin{tabular}{|c|c|c|c|c|}
\hline & $\begin{array}{l}\text { Slick A } \\
\text { CEWAF }\end{array}$ & $\begin{array}{l}\text { Slick A } \\
\text { HEWAF }\end{array}$ & $\begin{array}{l}\text { Artificially } \\
\text { Weathered } \\
\text { Source } \\
\text { CEWAF }\end{array}$ & $\begin{array}{c}\text { Artificially } \\
\text { Weathered } \\
\text { Source } \\
\text { HEWAF }\end{array}$ \\
\hline Naphthalene & - & 0.36 & 132.0 & 154.0 \\
\hline C1-Naphthalenes & - & 2.72 & 497.0 & 642.0 \\
\hline C2-Naphthalenes & 9.30 & 82.40 & 809.0 & 1110.0 \\
\hline C3-Naphthalenes & 13.40 & 197.0 & 589.0 & 837.0 \\
\hline C4-Naphthalenes & 7.56 & 169.0 & 349.0 & 505.0 \\
\hline Biphenyl & 1.60 & 1.91 & 66.0 & 87.50 \\
\hline Dibenzofuran & - & - & - & - \\
\hline Acenaphthylene & - & - & - & - \\
\hline Acenaphthene & 0.14 & 1.11 & 4.59 & 6.47 \\
\hline Fluorene & 2.17 & 14.9 & 51.4 & 69.2 \\
\hline C1 - Fluorenes & 4.93 & 61.7 & 119.0 & 168.0 \\
\hline C2 - Fluorenes & 7.08 & 105.0 & 179.0 & 253.0 \\
\hline C3-Fluorenes & 5.88 & 114.0 & 155.0 & 221.0 \\
\hline Anthracene & 0.10 & 2.04 & 4.62 & 6.17 \\
\hline Phenanthrene & 5.62 & 56.5 & 109.0 & 147.0 \\
\hline C1-Phenanthrenes/Anthracenes & 10.40 & 165.0 & 252.0 & 346.0 \\
\hline C2-Phenanthrenes/Anthracenes & 9.16 & 188.0 & 280.0 & 377.0 \\
\hline C3-Phenanthrenes/Anthracenes & 5.88 & 120.0 & 185.0 & 256.0 \\
\hline C4-Phenanthrenes/Anthracenes & 2.69 & 77.9 & 136.0 & 195.0 \\
\hline Dibenzothiophene & 0.97 & 8.59 & 19.1 & 27.0 \\
\hline C1 - Dibenzothiophenes & 2.55 & 36.9 & 66.5 & 88.3 \\
\hline C2 - Dibenzothiophenes & 3.15 & 65.6 & 99.1 & 133.0 \\
\hline C3 - Dibenzothiophenes & 2.46 & 51.9 & 74.4 & 103.0 \\
\hline C4 - Dibenzothiophenes & 2.12 & 29.7 & 41.9 & 63.0 \\
\hline Benzo(b)fluorine & 0.16 & 3.98 & 7.14 & 9.55 \\
\hline Fluoranthene & 0.09 & 1.44 & 2.26 & 2.86 \\
\hline Pyrene & - & 5.11 & 7.93 & 10.3 \\
\hline C1 - Fluoranthenes/Pyrenes & 0.82 & 21.0 & 36.2 & 48.8 \\
\hline C2 - Fluoranthenes/Pyrenes & 1.36 & 35.0 & 61.9 & 86.7 \\
\hline C3 - Fluoranthenes/Pyrenes & 1.49 & 43.1 & 81.8 & 106.0 \\
\hline C4-Fluoranthenes/Pyrenes & 1.21 & 32.8 & 58.9 & 80.1 \\
\hline Naphthobenzothiophene & 0.21 & 5.56 & 6.48 & 9.56 \\
\hline C1-Naphthobenzothiophenes & 0.71 & 21.6 & 28.4 & 41.8 \\
\hline
\end{tabular}




\begin{tabular}{lcccc} 
C2-Naphthobenzothiophenes & 0.80 & 25.6 & 35.1 & 50.3 \\
C3-Naphthobenzothiophenes & 0.52 & 20.0 & 25.6 & 45.1 \\
C4-Naphthobenzothiophenes & - & 9.2 & 15.7 & 22.8 \\
Benz(a)anthracene & 0.06 & 1.31 & 3.52 & 4.51 \\
Chrysene+Triphenylene & 0.67 & 17.2 & 17.4 & 25.2 \\
C1-Chrysenes & 1.17 & 35.9 & 49.2 & 68.3 \\
C2-Chrysenes & 1.16 & 37.9 & 60.6 & 90.8 \\
C3-Chrysenes & 0.67 & 23.9 & 44.4 & 68.8 \\
C4-Chrysenes & - & 17.3 & 38.8 & 53.8 \\
Benzo(b)fluoranthene & 0.06 & 2.1 & 2.2 & 2.9 \\
Benzo(j+k)fluoranthene & - & - & - & - \\
Benzo(a)fluoranthene & - & 0.35 & - & - \\
Benzo(e)pyrene & 0.10 & 3.52 & 4.05 & 6.20 \\
Benzo(a)pyrene & - & 0.47 & 1.03 & 1.26 \\
Indeno(1,2,3-cd)pyrene & - & 0.13 & 0.61 & 0.50 \\
Dibenz(a,h)anthracene & - & 0.67 & 1.32 & 1.56 \\
Benzo(g,h,i)perylene & 0.02 & 0.58 & 0.97 & 1.19 \\
\hline & & & & 6633.53 \\
Sum PAH & $\mathbf{1 0 8 . 4 4}$ & $\mathbf{1 9 1 7 . 9 4}$ & $\mathbf{4 8 1 0 . 1 3}$ & \\
\hline
\end{tabular}

Table S2

The individual PAH detection limits used in each stock analysis. Bold indicates one of the 14 photodynamic PAHs.

Water accommodated fraction stock $\mathrm{tPAH}_{50}$ composition $(\mu \mathrm{g} / \mathrm{L})$

\begin{tabular}{lcccc}
\hline & & & $\begin{array}{c}\text { Artificially } \\
\text { Weathered }\end{array}$ & $\begin{array}{c}\text { Artificially } \\
\text { Weathered } \\
\text { Source HEWAF }\end{array}$ \\
\hline Naphthalene & 0.012 & 0.024 & 0.12 & 0.12 \\
C1-Naphthalenes & 1.82 & 0.024 & 0.12 & 0.12 \\
C2-Naphthalenes & 0.012 & 0.024 & 0.12 & 0.12 \\
C3-Naphthalenes & 0.012 & 0.024 & 0.12 & 0.12 \\
C4-Naphthalenes & 0.012 & 0.024 & 0.12 & 0.12 \\
Biphenyl & 0.0096 & 0.0192 & 0.096 & 0.096 \\
Dibenzofuran & 0.422 & 3.92 & 13.1 & 16.4 \\
Acenaphthylene & 0.144 & 0.556 & 4.22 & 5.58 \\
Acenaphthene & 0.0176 & 0.0352 & 0.176 & 0.176 \\
Fluorene & 0.0152 & 0.0304 & 0.152 & 0.152 \\
C1 - Fluorenes & 0.0152 & 0.0304 & 0.152 & 0.152 \\
C2 - Fluorenes & 0.0152 & 0.0304 & 0.152 & 0.152 \\
C3 - Fluorenes & 0.0152 & 0.0304 & 0.152 & 0.152 \\
Anthracene & 0.0144 & 0.0288 & 0.144 & 0.144
\end{tabular}




\begin{tabular}{|c|c|c|c|c|}
\hline Phenanthrene & 0.02 & 0.04 & 0.2 & 0.2 \\
\hline C1-Phenanthrenes/Anthracenes & 0.02 & 0.04 & 0.2 & 0.2 \\
\hline C2-Phenanthrenes/Anthracenes & 0.02 & 0.04 & 0.2 & 0.2 \\
\hline C3-Phenanthrenes/Anthracenes & 0.02 & 0.04 & 0.2 & 0.2 \\
\hline C4-Phenanthrenes/Anthracenes & 0.02 & 0.04 & 0.2 & 0.2 \\
\hline Dibenzothiophene & 0.0152 & 0.0304 & 0.152 & 0.152 \\
\hline C1 - Dibenzothiophenes & 0.0152 & 0.0304 & 0.152 & 0.152 \\
\hline C2 - Dibenzothiophenes & 0.0152 & 0.0304 & 0.152 & 0.152 \\
\hline C3 - Dibenzothiophenes & 0.0152 & 0.0304 & 0.152 & 0.152 \\
\hline C4 - Dibenzothiophenes & 0.0152 & 0.0304 & 0.152 & 0.152 \\
\hline Benzo(b)fluorene & 0.0044 & 0.00664 & 0.0332 & 0.0332 \\
\hline Fluoranthene & 0.0176 & 0.0352 & 0.176 & 0.176 \\
\hline Pyrene & 0.277 & 0.028 & 0.14 & 0.14 \\
\hline C1 - Fluoranthenes/Pyrenes & 0.0176 & 0.0352 & 0.176 & 0.176 \\
\hline C2 - Fluoranthenes/Pyrenes & 0.0176 & 0.0352 & 0.176 & 0.176 \\
\hline C3 - Fluoranthenes/Pyrenes & 0.0176 & 0.0352 & 0.176 & 0.176 \\
\hline C4-Fluoranthenes/Pyrenes & 0.0176 & 0.0352 & 0.176 & 0.176 \\
\hline Naphthobenzothiophene & 0.0076 & 0.0152 & 0.076 & 0.076 \\
\hline C1-Naphthobenzothiophenes & 0.0076 & 0.0152 & 0.076 & 0.076 \\
\hline C2-Naphthobenzothiophenes & 0.0076 & 0.0152 & 0.076 & 0.076 \\
\hline C3-Naphthobenzothiophenes & 0.0076 & 0.0152 & 0.076 & 0.076 \\
\hline C4-Naphthobenzothiophenes & 0.0076 & 0.0152 & 0.076 & 0.076 \\
\hline Benz(a)anthracene & 0.0104 & 0.0208 & 0.104 & 0.104 \\
\hline Chrysene+Triphenylene & 0.0136 & 0.0272 & 0.136 & 0.136 \\
\hline C1 - Chrysenes & 0.0136 & 0.0272 & 0.136 & 0.136 \\
\hline C2 - Chrysenes & 0.0136 & 0.0272 & 0.136 & 0.136 \\
\hline C3 - Chrysenes & 0.0136 & 0.0272 & 0.136 & 0.136 \\
\hline C4 - Chrysenes & 0.0136 & 0.0272 & 0.136 & 0.136 \\
\hline Benzo(b)fluoranthene & 0.0092 & 0.0184 & 0.092 & 0.092 \\
\hline Benzo(j+k)fluoranthene & 0.01 & 0.02 & 1.13 & 0.875 \\
\hline Benzo(a)fluoranthene & 0.0044 & 0.00624 & 0.0312 & 0.361 \\
\hline Benzo(e)pyrene & 0.016 & 0.032 & 0.16 & 0.16 \\
\hline Benzo(a)pyrene & 0.0172 & 0.0344 & 0.172 & 0.172 \\
\hline Indeno(1,2,3-cd)pyrene & 0.0104 & 0.0208 & 0.104 & 0.104 \\
\hline Dibenz(a,h)anthracene & 0.01 & 0.02 & 0.1 & 0.1 \\
\hline Benzo(g,h,i)perylene & 0.0116 & 0.0232 & 0.116 & 0.116 \\
\hline
\end{tabular}

